\title{
Article \\ Tracking and Monitoring of the Alignment System Used for Nuclear Physics Experiments
}

\author{
Paul Nicolae Borza ${ }^{1, * \mathbb{D}}$ and Sorin Vlase ${ }^{2,3, *}$ \\ 1 Department of Computers and Electronics, Transilvania University of Brasov, Bulevardul Eroilor 29, \\ 500036 Brasov, Romania \\ 2 Department of Mechanical Engineering, Transilvania University of Brasov, Bulevardul Eroilor 29, \\ 500036 Brasov, Romania \\ 3 Romanian Academy of Technical Sciences, Bulevardul Dacia 26, 030167 Bucharest, Romania \\ * Correspondence: borzapn@unitbv.ro (P.N.B.); svlase@unitbv.ro (S.V.); Tel.: +40-722643020 (S.V.)
}

Citation: Borza, P.N.; Vlase, S. Tracking and Monitoring of the Alignment System Used for Nuclear Physics Experiments. Symmetry 2022, 14, 47. https://doi.org/10.3390/ sym 14010047

\section{Academic Editor: Giuseppe Bagliesi}

Received: 19 September 2021

Accepted: 14 December 2021

Published: 31 December 2021

Publisher's Note: MDPI stays neutral with regard to jurisdictional claims in published maps and institutional affiliations.

Copyright: (c) 2021 by the authors Licensee MDPI, Basel, Switzerland. This article is an open access article distributed under the terms and conditions of the Creative Commons Attribution (CC BY) license (https:// creativecommons.org/licenses/by/ $4.0 /)$.

\begin{abstract}
The ELI-NP (Extreme Light Intensity-Nuclear Physics) project, developed at the Horia Hulubei National Institute for RD in Physics and Nuclear Engineering (IFIN-HH), has included one component dedicated to the study of interactions between brilliant gamma-ray and matter, with applications in nuclear physics and the science of materials. The paper is focused on the interaction chamber, an important part of the facility which hosts the experiment's samples. The interaction chamber is endowed with a mobile sample support (holder), which automatically tracks the $\gamma$-ray beam. The $\gamma$-ray radiation source presents a slight variation of the direction of the emitted radiation in time. The built system ensures the permanent collimation between the $\gamma$-ray beam and the sample that is being investigated. This is done with two electric motors, which have a symmetrical movement with respect to the center of a rectangle. The specific measures taken by the design and implementation that permit to reach performances of tracking system are emphasized in the paper. The methodology considers the relative displacement between the detectors with which the laboratory is equipped and the absolute position in space of the sample boundary. The control of this motion is designed to respect the symmetry of the system. Both facets of the project (hardware and software) are detailed, emphasizing the way in which the designers ensured compliance with the system of real-time operation conditions of the tracking and monitoring system.
\end{abstract}

Keywords: dynamic behavior; interaction chamber; control; automatic alignment

\section{Introduction}

The $\gamma$-ray beam system under construction at ELI-NP provides a very bright photon beam with unprecedented bandwidth and tunability. To fully take advantage of this performance, a number of interesting physics cases were identified in the WhiteBook of the ELI-NP project and then detailed in the Technical Design Reports [1-4]. Nuclear instrumentation is used to highlight an important process regarding the reactivity of matter in its interaction with the $\gamma$-ray. The ELI-NP [1] facility allows the investigation of nuclear phenomena by colliding photons ( $\gamma$-ray radiation) with different samples of matter. This occurs inside an interaction chamber (ICh), where a medium vacuum $\left(10^{3} \mathrm{~Pa}\right)$ is realized. The facility includes the $\gamma$-ray source (20 MeV pulsed) placed on a vibration-free platform and several test benches placed on the $\gamma$-ray beam trajectory. The interaction chamber is just one of the facilities that benefit from the radiation source, and it is placed in the path of the $\gamma$-ray beam.

The proposed system described in this paper tries to answer the challenges of this new way proposed for the investigation of matter. The paper identifies the relevant features that are considered in the design and testing phase. There are mechanical factors related to the initial alignment procedure and potential relative variation of position between facility elements: vibration that could appear as results of the particular geographic seismicity or 
due to anthropogenetic activities; the structural behavior of the ELI-NP assembly; and the dynamics of the acting system used for displacement of the samples holder inside the ICh.

Ultrasensitive detectors, ELIADE (ELI-NP array of detectors), are placed around the ICh. They are used to highlight the reactions that occur between the $\gamma$-ray and the materials used as samples [5-10].

The bright $\gamma$-ray source is based on Compton incoherent inverse scattering of a highfrequency, low-emission pulsed laser light that collides with a relativistic electron beam with energies of up to $720 \mathrm{MeV}$. The generated photons have controllable energies that can reach up to $20 \mathrm{MeV}$ [11-15]. The direction in which they are emitted may vary slightly over time $[16,17]$. Thus, it is necessary that the test benches that use the $\gamma$-ray flux as excitation remain during the experiments in the path of the generated $\gamma$-ray beam [18-22]. In order to ensure the collision of the radiant beam with the nuclei of the investigated samples, it is necessary to maintain the sample holder in the way of the $\gamma$-ray radiant beam.

Between the $\gamma$ beam and the holder or support of the samples with which it interacts, there is a support system that can move in the plane perpendicular to the beam. This drive system has stepper motors and mechanisms adapted for vacuum conversion of the rotational movement of the motors in the translation of the pistons of the respective mechanism.

The tracking system is coordinated by a personal computer (PC) that controls the image acquisition from a high resolution and precision sCMOS camera. Using a scintillator, the high energy image projected on the scintillator is converted into a fluorescence image that is projected onto the digital camera. The camera parameters, integration time, region of interest, the data flow rate on USB interface, etc. are adjusted automatically in order to adapt these with the tracking system demands. The resolution of the tracking system depends on the magnification factor provided by the scintillator together with the optical system and sCMOS camera. Pattern recognition software provides the dynamic reference point used by the tracking system $[19,20]$.

The image acquired is correlated with the signals provided by photo-multipliers. At the initial moment, the experiment was settled by arranging manually, using a visible optical laser system (see Figure 1), the position of the sample holder relative to the detectors.

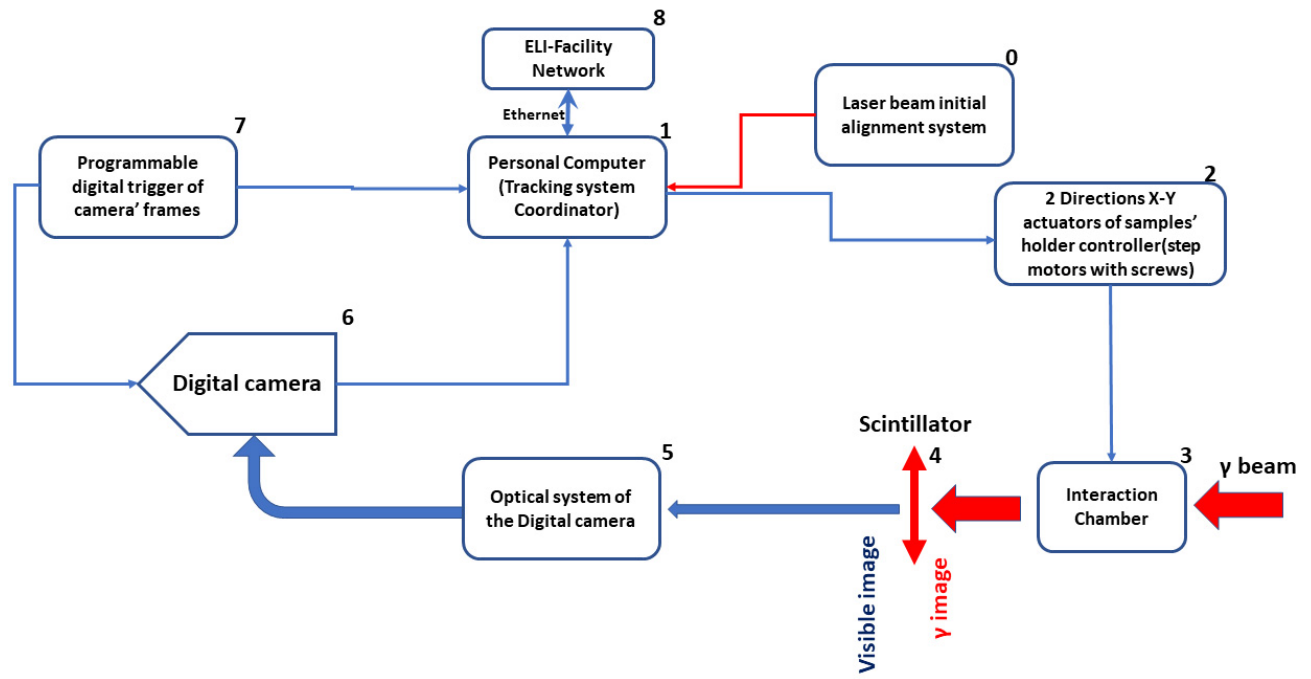

Figure 1. Block diagram of the ELICAMM-GAMMA tracking system. 0-laser beam (visible) for initial alignment of ICh; 1-the main element of the tracking system (personal computer); 2-direction positioner controller for the 2-axis step motors; 3-interaction chamber with sample holder; 4 -scintillator places on transmitted $\gamma$ beam; 5 -optical system including a 45-degree mirror (visible); 6-high resolution digital camera; 7-programmable digital frame trigger for image acquisition from digital camera; 8-ELI facility Ethernet network. 
The paper reveals how the authors solved the challenges related to precision, accuracy and real-time constraints regarding the tracking functions attached to the interaction chamber.

The aspects related to the computer programming that ensures the overall control of the system are highlighted, and the validation aspects relative to the actuating component are detailed. The results of the tests performed on the actuating system are given. In conclusion, the system performance that allows the full satisfaction of the integration requirements in the ELI facility is highlighted. The test results serve as proof of the accuracy in positioning obtained with the designed solution and also its adequate speed that allows us to consider the tracking system as working in "real time".

The tracking system consists of the scintillator (4), the optical projection system of the scintillator for the digital sCMOS camera, jointly mounted on the external wall of the interaction camera, as well as the image processing (computer) and control elements (drivers and linear stages) of the actuators related to the sample holder. An important challenge of this system is to achieve precise alignment between the target and the $\gamma$-ray beam on a variable distance of $10-100 \mathrm{~m}$. The size of the target is assumed to be $3 \mathrm{~mm}$. In order to realize this goal, we assume a cylindrical ICh with a $175 \mathrm{~mm}$ diameter. The alignment system is inserted inside this chamber. The holder is brought in the fixed plane established for experiments. This is correlated with the fixed position of the detectors and thus ensures the repeatability of the measurement conditions. The ICh is emptied to avoid possible noises produced by the interaction of $\gamma$-ray radiation with the gases in the chamber. The target, which must be supported by the system, may be solid, gaseous or liquid.

The correlation between the footprint of the $\gamma$-ray beam on the perpendicular plane containing the sample and the signals captured by ELIADE detectors is important for the accuracy of all experiments. This is one of the reasons for which the tracking system must accurately follow the $\gamma$-ray beam direction variation in time. In fact, the tracking system must respond at least four times as fast as a maximum of the time variation of the $\gamma$-ray direction (condition imposed by contractor).

Related to the "real time feature", we evaluated experimentally the delays due to signal propagation but also those due to its processing on the different stages. To cover this aspect, several methodologies for processing were evaluated. It is necessary to mention that the conception of the tracking system (TS) has to take into account potential new configurations that could be adapted in the future.

In connection with the optical system, the correlation between the scintillator and its quantum efficiency and the performance of the image acquisition sensor are essential. Regarding the latter aspect, CCD (charge coupled device) and sCMOS (scientific complementary metal-oxide-semiconductor) sensors were analyzed. The quantum efficiency, the storage capacity of the charge for each pixel, the "noise" (thermal electrons produced in operation at a certain temperature) and the scanning system of the sensor matrix as well as the maximum scanning frequency were considered. Finally, an sCMOS sensor was chosen that ensures an acceptable compromise between the quantum efficiency and image acquisition speed as well as the resolution and sensitivity of photo-sensitive cells. Inside the ICh, there is a target holder system, used for the experiments at the $\gamma$-ray beam systems at ELI-NP [23].

From the point of view of the actuation system, the solution ensures a maximum resolution in positioning in loop (1.25 um), so the theoretical analysis and the practical implementation aimed at maintaining the nominal limits of the resistant torque in order to avoid the loss of actuation steps.

Mainly, the design considered changes of the scintillator, shape and material of the sample, and methodologies for the processing of signals in order to preserve the quality of the tracking system. As result, we describe in the paper not a simple embedded solution, but in fact a software framework that can be easily adapted for the futures changes that could appear. The software, designed to realize the control of the actuators, uses the symmetry of the rectangle work area. 


\section{Design and Implementation of the Tracking System}

Several potential solutions with their advantages and disadvantages were analyzed. After a study of the constraints of the alignment system, its structure and mechanics, two challenges were identified.

The first of the challenges is to find a way to identify from the analysis of the projection of the image of the sample holder (in the $\gamma$ field) a point or landmark shape. Thus, a stable optical representation of the $\gamma$ beam is obtained, and its possible displacements can be, from the analysis of the images acquired successively in relation to time, determined.

The second challenge is to find technical solutions that allow minimizing errors in the positioning of the sample holder when it follows the variation of the direction of the $\gamma$ beam incident to the interaction camera.

Another important aspect that had to be taken into account was the requirement to use for the system manager a PC running the Windows operating system which was not designed for real-time applications and does not guarantee perfectly synchronous execution of the tasks that implement the functions of the tracking system.

Regarding the first challenge, we further detail the way in which the design and particular implementation of the optical and electronic systems allowed to obtain the desired accuracy in following the direction fluctuations of the $\gamma$ beam.

In the placement phase of the components used by the ELI facility, a laser alignment system allowed us to initially align all the components of the system. Considering the attenuation produced when crossing the sample support placed in the ICh by the $\gamma$ beam, its image was projected on the surface of the scintillator that we placed outside the ICh. The designed optical system includes the scintillator, with the role of converting the image of the incident $\gamma$ photon flux into a visible flux. As the electronic instrumentation is significantly affected by the $\gamma$ photon flux, the image acquisition system was placed outside the direct beam. In this sense, the image obtained on the scintillator was deflected at 90 degrees using a mirror inclined at 45 degrees relative to the main optical axis. The optical axis is perpendicular to the scintillator surface. Thus, the image formed on the scintillator was projected using a group of lenses into the plane of the digital camera sensor. In our case, the digital camera sensor was equipped with a sCMOS sensor. This represents the main processing system component dedicated for image acquisition and processing, but also for the command and control of the system' actuators. The programs were developed in order to implement algorithms capable of quickly scanning the digital camera and detecting on the images generated by the scintillator their characteristics patterns and features. The obtained image has a resolution of 16 bits; respectively, it can highlight up to 65,536 in distinct shades. In the tracking process, the successive images' patterns and their features are used as landmarks necessary to follow how the gamma beam is changed in time (inherent variation due to ELI gamma source. The developed programs allow to detect the pattern characteristic of the image of the sample support, and thus this became the reference element, or landmark, in relation to which the tracking system became operational.

The software developed implemented a workflow in accordance with the developed methodology as illustrated in Figure 2.

This algorithm commands periodically the acquisition of images from the digital camera. The successive acquired images are compared in order to detect the possible relative displacement of the beam. The system is aligned when a minimum of three successive images indicate the same position of the settled landmarks. The tracking system has three linear actuators equipped with stepper motors. All are positioned inside the ICh. Two motors are placed orthogonally, perpendicular to the optical axis of the system, and the third motor has the role of allowing the manipulation of samples, respectively, the movement parallel to the optical axis of the sample holder, thus facilitating the manipulation of experimental samples. A three-channel electronic controller is used in conjunction with the appropriate controllers for controlling the stepper motors. This controller is subordinated to the personal computer and communicates with it through command-andcontrol telegrams. 


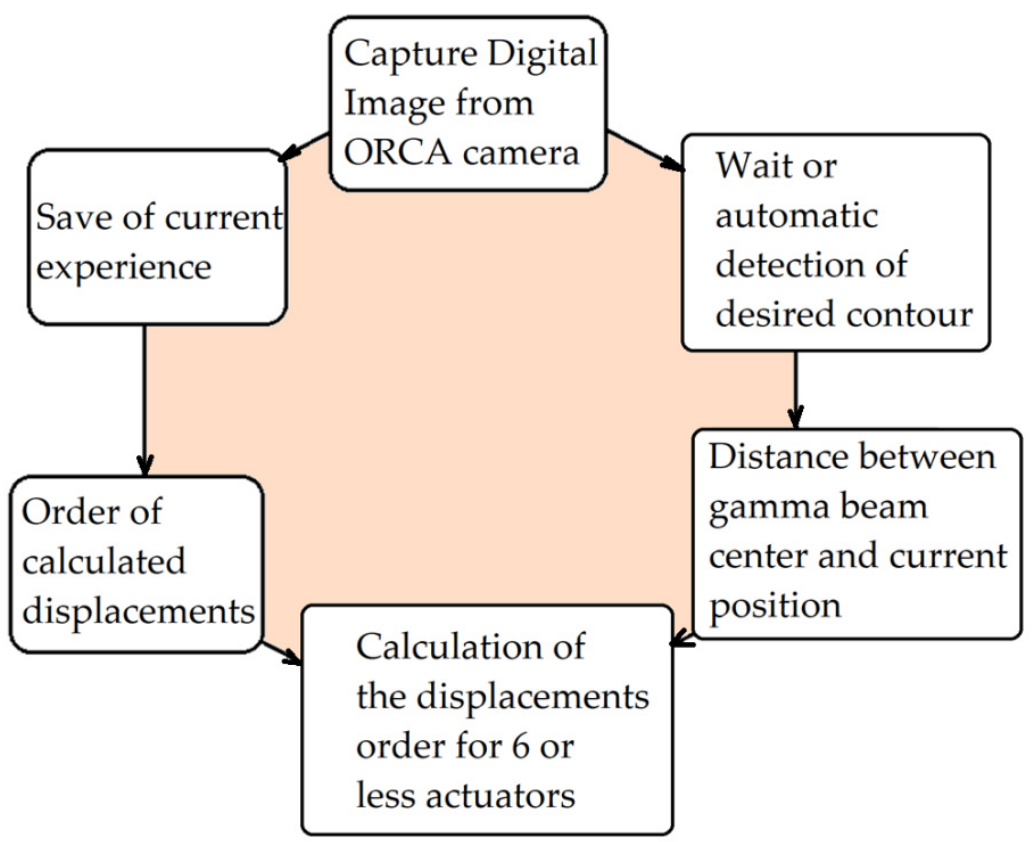

Figure 2. Main workflow diagram of the ELICAMM-GAMMA system.

The optimization of the alignment system involves the tracking system firmware that should include the important features as follows:

- Automatic detection of shapes into the acquired images and features related user interface for indicating the region of interest;

- Automatic tracking based on image features detection;

- Manual and automatic change of optical integration time using the facilities of the camera (front and level detection).

To obtain this, procedures and tests are made to illustrate the specific and general performances of implemented ICh tracking system (Figure 2).

For this study, it is necessary to know the geometry of the test bench (dimensions, environmental conditions, and electromagnetic and radiation shielding of measurement system), the optics (from beam generation to ICh and target) and the $\gamma$-ray beam system of detection (scintillator, optical system, capture and processing of the image, and control of positioning system).

\section{Constructive Interaction with Camera Alignment System against a $\gamma$ Beam}

The tracking of the $\gamma$ flow inside the interaction chamber was performed by displacement of the sample holder, which is automatically operated by two stepper motors placed orthogonally.

Particular attention was paid to the component corresponding to the drive system for the stepper drive motors used, and that assured the X-Y displacement of the samples' holder on the perpendicular plan to the main axis of the interaction chamber. The actuation was implemented in an open loop, taking into account the constraints due to the placement of the actuating elements in the $\gamma$ radiant field, as well as the requirements to ensure a high resolution and repeatability in the positioning of the sample holder. The implementation of a closed-loop solution would have involved the placement of position sensors inside the interaction chamber, their electronic components being able to be significantly influenced by $\gamma$ radiation.

In order to respond to the second challenge, it was decided to develop a separate system for testing and validating the actuation within the tracking system. The implemented solution is illustrated by the block diagram in Figure 3. 


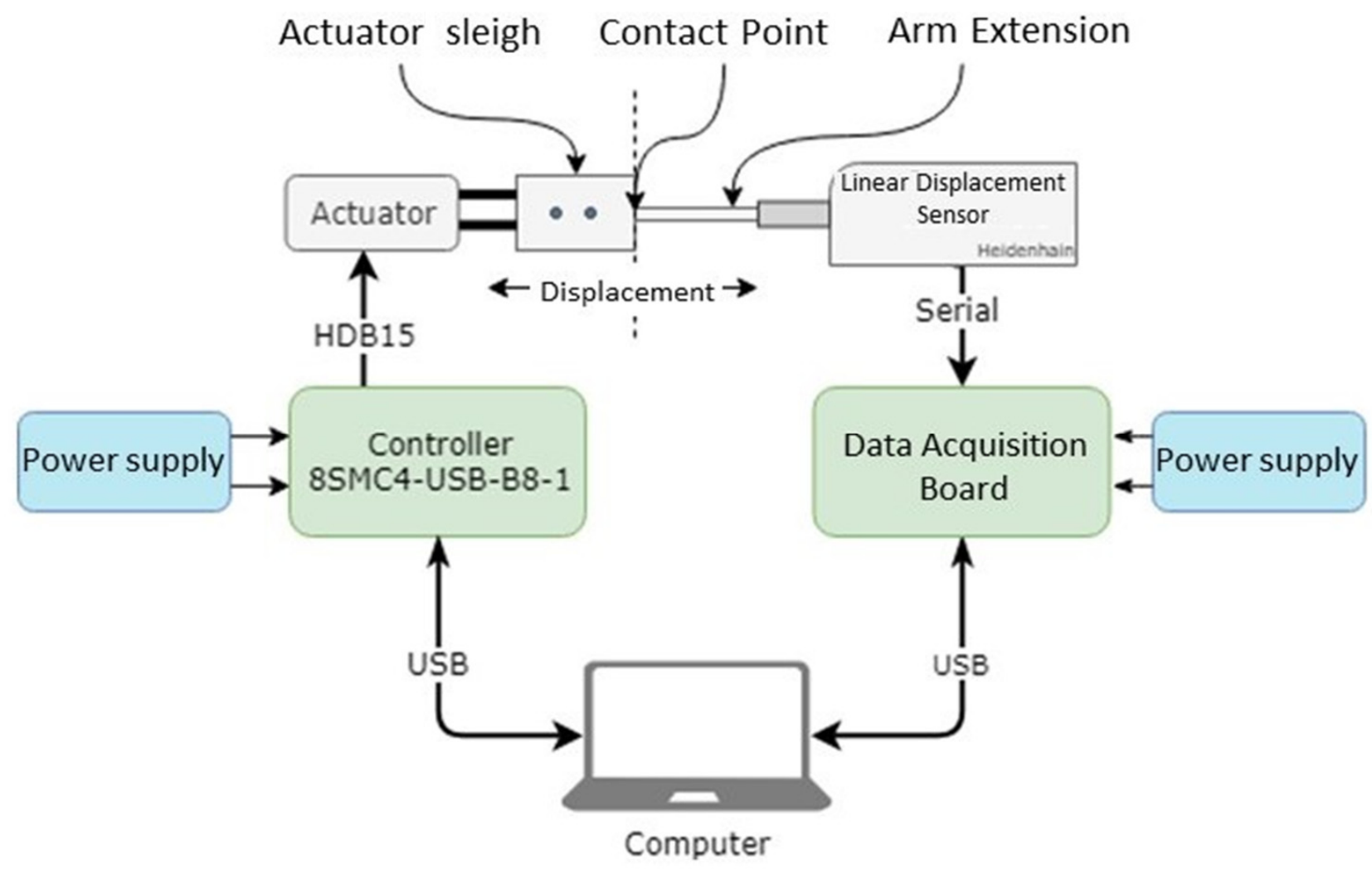

Figure 3. Block diagram of the test bench used for validation of one-axis positioning system (component of ELICAMM-GAMMA system).

As illustrated in Figure 3, the developed test subsystem was based on a solution similar to that used in the tracking system; the personal computer ensured the control by a serial interface. This interface was used to transmit telegrams to the electronic controller of the step motor.

The axis of the actuator was aligned with the axis of the displacement sensor, data provided by it being also processed by the personal computer.

The lubrication of the drive mechanism of the motors can influence the accuracy of movement when the step motor is controlled in the open loop. Additionally, the speed profile generated by the electronic controller of the actuator can generate positioning errors. In relation to the required displacement, the speed profile must also be adapted so as to minimize both displacement errors and execution time.

Through the program used for the control of the drive system, the drive component was tested, having as control parameters the prescribed travel, the adopted speed profile, as well as the mechanical load that the system supported. The direction of travel was reversed after each displacement performed, thus generating a round trip cycle for the tested actuator.

\section{Validation of Real-Time Operation for the Tracking System}

The most important effort was put toward developing and testing the software necessary for the control of the whole system. Using Visual Studio 2017, the algorithms for acquired image processing, communication and the accuracy in control of the quadrature settled actuators were developed and tested on components. In parallel, a test bench necessary for the validation and fine adjustment of the actuator's controller was implemented and used for testing of the acting system linked with the samples' holder. The ICh was mounted in the ELI laboratory facility. The ICh sealing system was tested for medium vacuum level, and the tests proved the appropriate mechanical design and accurate mechanical execution of the ICh. 
The ICh was clamped on two planes where two actuators assure the movement on the orthogonal plane $(X-Y)$ on the brilliant $\gamma$-ray beam of the samples' holder placed inside the ICh. The kinematics of the acting system was designed, simulated and experimentally validated. An independent laser beam alignment system was used to establish the initial 'reference position'. This assured the raw alignment of the ICh. Following the analysis of possible events during image acquisition and processing, the transfer to the computer and the recognizing of the landmark shape, scaling the landmark position and converting the coordinate difference in steps for the stepper motors were performed.

To assure appropriate control of the whole tracking system, the image acquisition performance was tested and validated. A report with the data obtained as a result of the tests performed helped the team in fine adjustment of the control system parameters. The tests were focused on the image acquisition time, local image processing by the camera and the transfer of the above image for the computer. The period of time necessary for the abovementioned processing stages was considered during the design of the system software. The initial software routine was improved, and automatic and manual triggering of image acquisition were calibrated and validated. The software implements two loops:

i. Implementation of the image acquisition;

ii. Detection of the target for the $X-Y$ linear stage that is activated with the updated target value.

The operating system used was Windows 10, which does not guarantee an exact time of execution for each thread. The experimental results show that the whole loop in control had a period of less than $100 \mathrm{~ms}$ (10 sps), in accordance with the potential slight variations of the brilliant $\gamma$-ray beam direction produced by the ELI source of radiation.

The image acquisition can be controlled by the software loop, or it is possible to be triggered on the mother frequency produced by the ELI system; a very precise hardware delay generator was used for triggering the image. The measuring head was precisely aligned with the step-by-step actuator. A series of tests were performed. The results showed that for different mechanical loads in the range of $1-3 \mathrm{~kg}$, the motor-driven slider can have systematic errors in the range of 0 to 3 microns [23]. These errors are much smaller than the errors accepted by the application. The load and the maximum speed as well as the speed variation slope were changed. In all experimental cases, it was found that the errors in positioning were maintained within the limits specified above. The step-by-step motor drive system had an open loop resolution of $1.25 \mathrm{um}$. In order to assure high precision of the acting system, we conceived the experimental test bench. We should mention that in the case of our application, the mechanical load (mass operated) was relatively constant with below $1 \mathrm{~kg}$ carried mass. We considered it necessary to test the accuracy of the control system for different movement speeds (between 1000 and 2000 steps per second, respectively, $1.25 \mathrm{~mm} / \mathrm{s}$ or $2.5 \mathrm{~mm} / \mathrm{s}$ ) and for different loads (0 kg to $2 \mathrm{~kg}$ ) applied to the system.

In Figure 4, on the axis of the abscissa, we calculated the number of consecutive cycles (go N steps and return the same number of steps), and on the ordinate axis, we show the error measured on the experimental stand. The figure illustrates the results of the tests carried out when the controlled movement is oscillating between $+/-200$ to $+/-3000$ steps, at different loads applied to the mechanism. The results show an error of less than 6 steps $(7.5 \mu \mathrm{m}$, respectively) in any of the situations tested. The results obtained are more than satisfactory.

Special attention was paid to the analysis of the potential sources of errors in the detection of the $\gamma$ beam (sensors, conversion of $\gamma$ beam in a beam in the visible spectrum, data or/and camera acquisition system, control of alignment system and acting system that must assure the precise positioning of IC in the $\gamma$ beam direction). In addition, the methodology and afferent algorithms for alignment system were analyzed for proposing three different methods for the detection of region of interests (ROI) on the captured image.

Going forward with the analysis, the paper reports the possibilities to use appropriate solutions for the conversion of the $\gamma$-ray "image" into a visible image using adequate 
scintillators. The BGO scintillators are considered adequate for this application. Special attention was paid for the simulation of the interaction between the $\gamma$-ray beam and the scintillator material. In addition, market analyses were conducted in order to find out the potential appropriate digital camera susceptible to be used for image acquisition and for the afferent optical system. In this sense, we considered a sCMOS camera with excellent features related to SNR, sensitivity, resolution and triggering features. For the manipulation of the sample holder, a precision acting system that can be operated in vacuum was tested and validated.

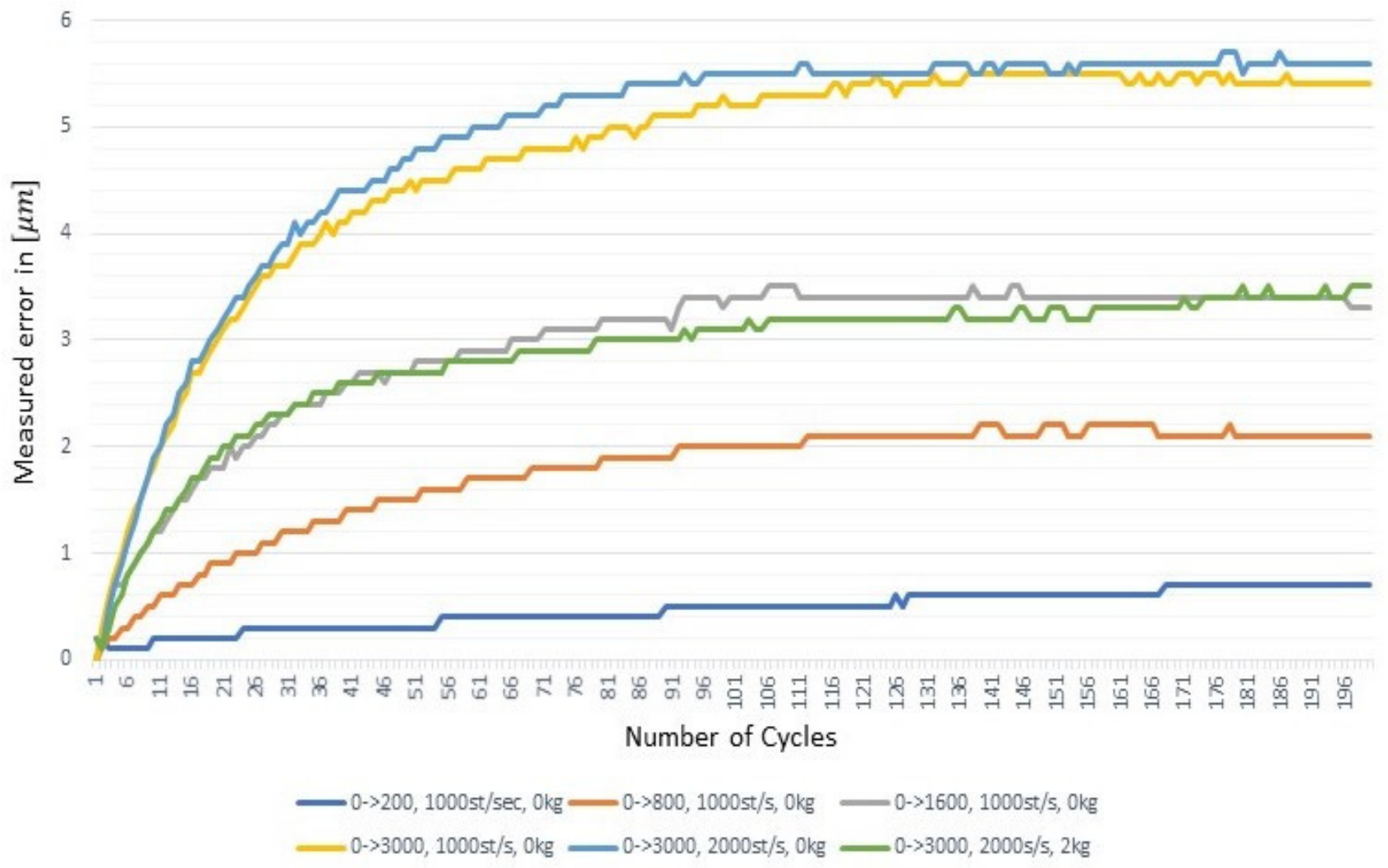

Figure 4. Experimental data: The abscissa axis illustrates the number of complete back and forth cycles performed, and the ordinate axis illustrates the positioning error measured experimentally in microns when moving $2 \mathrm{~kg}$.

All the software components were integrated. For example, the calibration facility, the images analysis methodologies, the manual and automatic adaptation of sampling rate, and other parameters of the sCMOS camera. All these features and many others were optimized and integrated into the final version of the software. An image of the program user interface is illustrated in Figure 5.

The capturing of the image by digital camera was synchronized with the acquisition of the main signal provided by the ELI system to avoid the errors related to the sampling period in the image acquisition.

Related to the mechanics of the ICh, significant technological difficulties occurred, related to the high flexibility of the ICh body. The mechanical response was obtained using CAD software. 


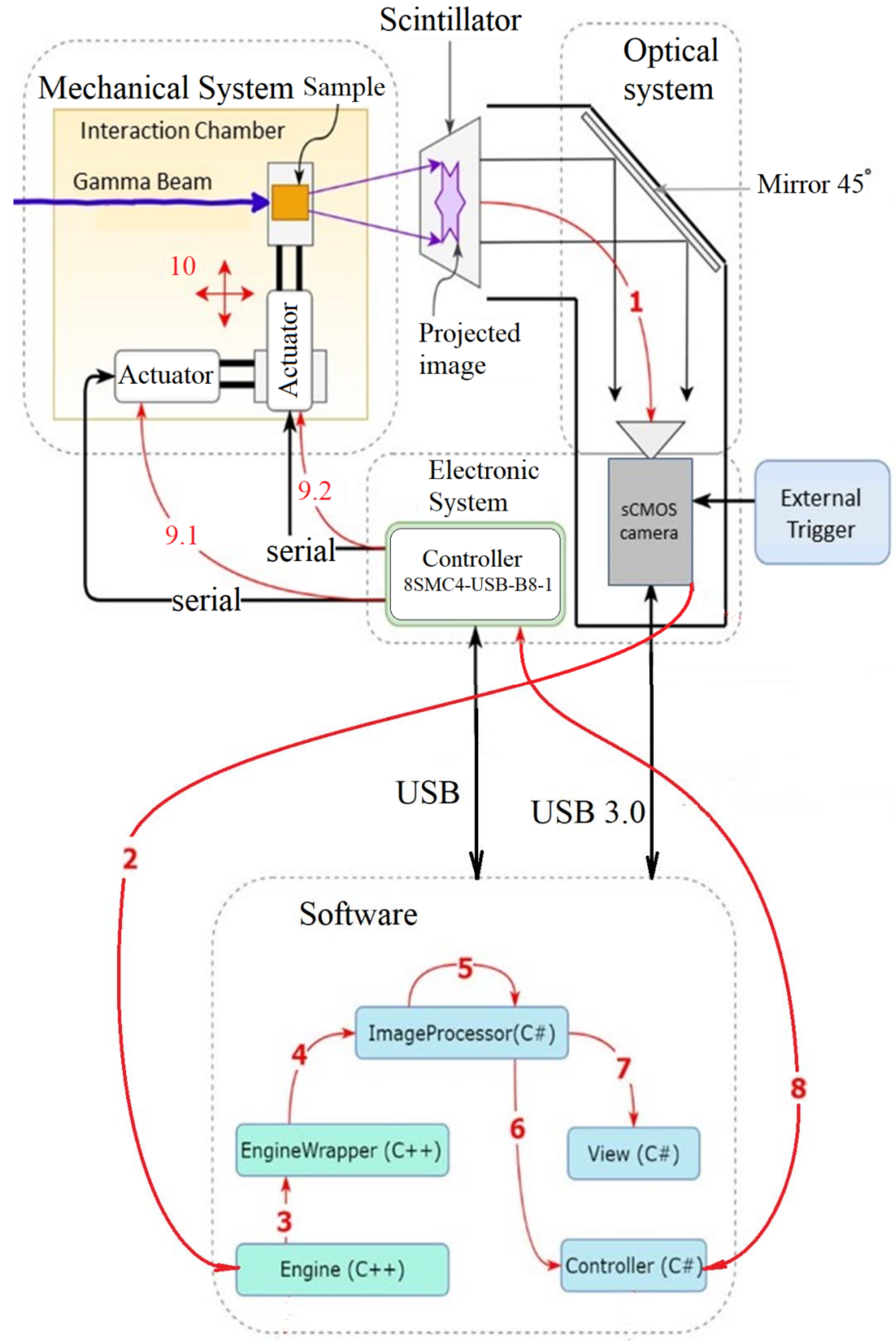

Figure 5. Detailed block diagram of tracking system (ELICAM-GAMMA). Hardware and software components, interfaces and software components that are involved in data processing. 
Figure 5 shows the ways corresponding to the production of both physical and logical signals that correspond to the program components involved in signal processing. The elements that process the signals and the signal delays due to their propagation or processing are identified. A first delay is due to signal conversion due to the scintillator. It generates an image of the scene corresponding to the objects in the interaction camera, mainly the sample holder and the sample itself, in an image in the visible domain. The photons in the visible range thus generated are captured by the sensitive cells of the image sensor of the ORCA Flash 4.0 digital camera (1). The sampling period, but also the triggering of this camera, are controllable by program. Additionally, through the program, we can control the way of scanning the image sensor cells and we can choose the appropriate operating mode. The image stored in the digital camera's memory is transferred serially via USB3.0 to the personal computer (2) (grabbing process). Using components of the DCAM library, we implemented image capture, primary processing of related data, and format conversions from RAW (minimal pixel data processed) to a BMP format, which become a device independent bitmap file format $(3,4)$. The resolution of the acquired image is 16 bits, in shades of gray, and the image thus obtained is processed by applying static or dynamic methods (depending on the choice made by the user) in order to highlight the contours from the image. Thus, the object that is considered the landmark that is used in the process of tracking the $\gamma$ radiant flux is determined (5). On the one hand, the processed image is displayed in real time on the screen of the personal computer, and on the other hand, it is used, by identifying the established landmark, to calculate the eventual displacement that the incident $\gamma$ flow to the interaction camera suffers during the sampling period. In the latter case, the computer scales the newly obtained reference data and calculates accordingly the number of steps in the $\mathrm{X}$ direction, respectively, in the $\mathrm{Y}$ direction, which the stepper motor has to execute (6). The steps to be executed, the adequate speed profile suitable for the movement to be executed as well as all the status information of the $2 \mathrm{D}$ drive (two dimensions) are transferred serially via the USB3.0 interface (8) to and from the two-channel electronic controller that controls the stepper motors. The electronic controller, in turn, outputs the control signals for the stepper motor via its power interfaces (six wires corresponding to the phases of the step motor). Figure 6 illustrates the image of the user interface with the buttons and windows needed to control the system, but also with the frames used to view images in real time.

The grabbing, processing and post-processing of images captured from sCMOS camera were identified, and the time necessary to execute the above-mentioned routines was measured. All of these were determined in the context in which Windows 10 runs a varied palette of programs that induce specific delays.

The experimental results show that the whole loop in control has a period less than $100 \mathrm{~ms}$ (10 sps), in accordance with the potential slight variations of the brilliant $\gamma$-ray beam direction produced by the ELI source of radiation.

Going forward with the analysis, the paper investigated the possibilities to use appropriate solutions for the conversion of the $\gamma$-ray "image" into a visible image using adequate scintillators. The BGO scintillators are considered adequate for this application. Special attentions were paid for the simulation of the interaction between the $\gamma$-ray beam and the scintillator material. Additionally, market analyses were conducted in order to find out the potential appropriate digital camera susceptible to be used for image acquisition and for the afferent optical system. In this sense, we considered an sCMOS camera with excellent features related to SNR, sensitivity, resolution and triggering features. For the manipulation of the sample holder, a precision acting system that functions in vacuum was tested and validated.

The capturing of an image by digital camera was synchronized with the acquisition of the main signal provided by the ELI system to avoid the errors related to the sampling period in the image acquisition. 


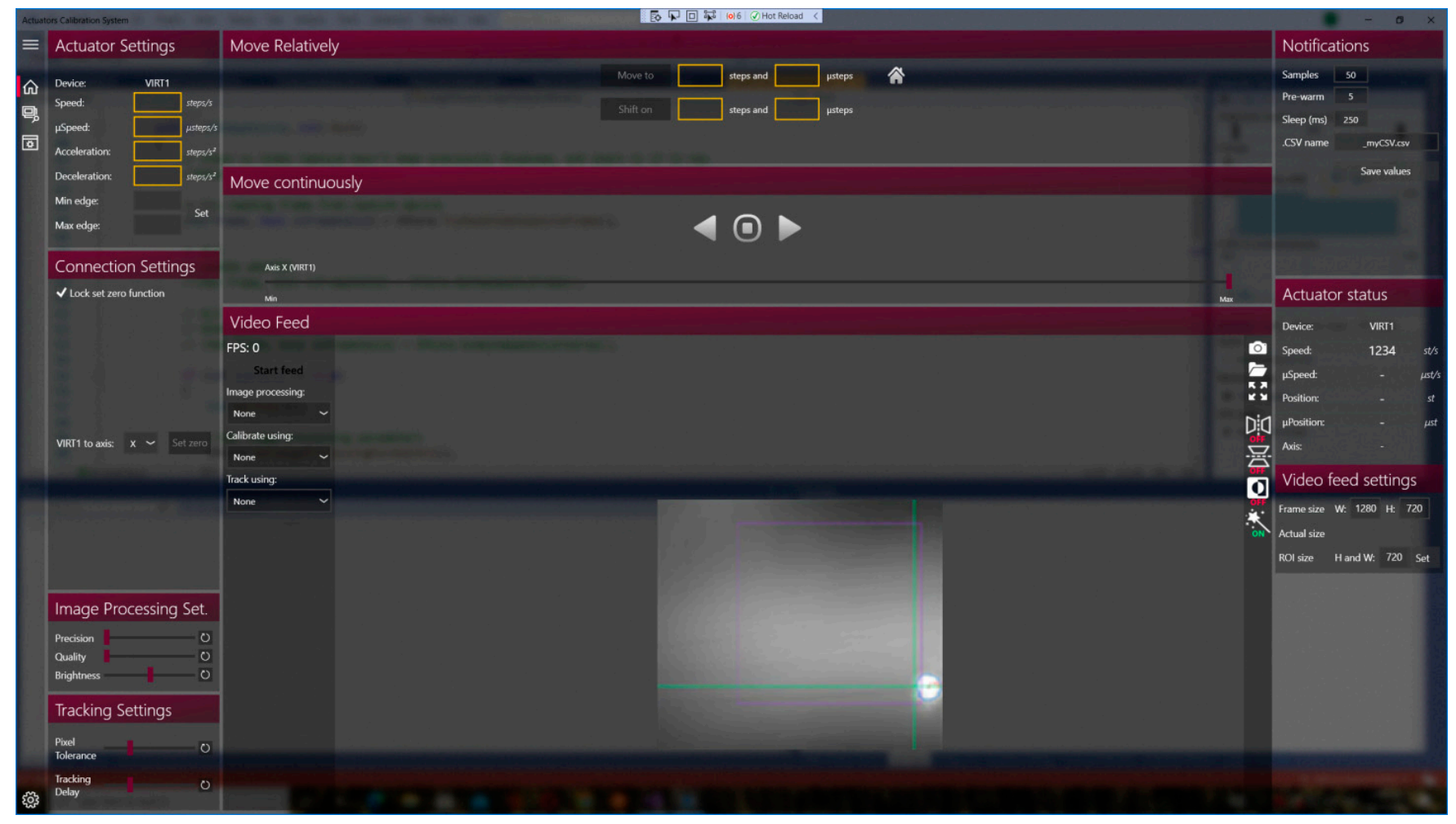

Figure 6. User interface of the tracking system, includes the control parameters: actuator speed profile, the increments to do, the serial interface connection settings, the threshold, quality, brightness of image captured, the video feed parameters and also the control buttons for manual displacement of acting motors, and the status of thewhole system.

\section{Discussion and Conclusions}

The paper presents how the authors solved the problem and challenges related to the determination of the relative displacement that appears during experiments with the interaction chamber in the ELI facility between the $\gamma$ beam and the sample holder.

The tracking system dedicated to the tracking of the $\gamma$-ray beam for the samples' holder placed inside the interaction room consists of the following:

(i). A detection part necessary to sense the slight direction variation of the $\gamma$-ray beam;

(ii). A set of linear stages in quadrature (step-by-step motors and the mechanical components for conversion of the motor rotor in a linear displacement) that ensure the tracking of the $\gamma$-ray beam by the samples' holder in a perpendicular plane on the $\gamma$-ray beam;

(iii). The dedicated electronic system that implements the processing of input signals and generates the control messages for the linear stages.

The constructive solutions were chosen by taking into account the optimization of operations during the aligning process but also the $\gamma$-beam pulse duration. We proposed eight digitally controlled actuators with spherical probes for the support chamber of the target. The system operates as follows: after the initial pre-alignment using an additional laser system, we save the obtained settings, which then become the reference for the automatic adjustment of alignment system. The eight actuators are controlled so that they reach the calculated target points in accordance with the geometric model of the plant. Meanwhile, the mirror at $45^{\circ}$ (see Figure 6) automatically adopts the position of the reflecting beam obtained by using the scintillator. In this way, the system captures the image of the $\gamma$-ray radiation spot and allows an automatic adjustment of the target inside the ICh. If this is not possible at the time, in order not to endanger the project, testing in a laboratory abroad that provides a $\gamma$-ray beam system is envisaged. Currently, we take 
into consideration three locations where this can be achieved. The total delay produced during acquisition, the processing of images and transferring of serial commands to the step motor controller is below $180 \mathrm{~ms}$ in all cases. This means that the system fully satisfies the functional constraints related it capability to track the $\gamma$-beam position variations in time. Additionally, the authors investigated how the operating system (Windows 10) affects the tracking functionality. The system responds correctly in a large set of use cases tested.

In the final phase, we aim for the experimental validation of the designed and built systems. We analyzed how the system meets the requirements and how this will be implemented in the experiments at ELI-NP.

Author Contributions: Conceptualization, P.N.B. and S.V.; methodology, P.N.B.; validation, P.N.B. and S.V.; formal analysis, P.N.B. and S.V; investigation, P.N.B.; resources, P.N.B. and S.V; data curation, P.N.B.; writing—original draft preparation, P.N.B.; writing-review and editing P.N.B. and S.V; visualization, P.N.B. and S.V; supervision, P.N.B. and S.V.; project administration, P.N.B. and S.V.; funding acquisition, P.N.B. and S.V. All authors have read and agreed to the published version of the manuscript.

Funding: This research was funded by National Institute for Nuclear Engineering, grant 16/2016 and the APC was funded by Transilvania University of Brasov.

Institutional Review Board Statement: Not applicable.

Informed Consent Statement: Not applicable.

Data Availability Statement: Not applicable.

Acknowledgments: We want to thank the reviewers who read the manuscript carefully and proposed pertinent corrections that led to an improvement in our manuscript.

Conflicts of Interest: The authors declare no conflict of interest. The funders had no role in the design of the study; in the collection, analyses, or interpretation of data; in the writing of the manuscript; or in the decision to publish the results.

\section{References}

1. Habs, D.; Tajima, T.; Zamfir, V. Extreme light infrastructure-nuclear physics (ELI-NP): New horizons for photon physics in Europe. Nucl. Phys. News 2011, 21, 23-29. [CrossRef]

2. Gales, S.; Tanaka, K.A.; Balabanski, D.L.; Negoita, F.; Stutman, D.; Tesileanu, O.; Ur, C.A.; Ursescu, D.; Andrei, I.; Ataman, S.; et al. The extreme light infrastructure-Nuclear physics (ELI-NP) facility: New horizons in physics with $10 \mathrm{PW}$ ultra-intense lasers and $20 \mathrm{MeV}$ brilliant gamma beams. Rep. Prog. Phys. 2018, 81, 094301. [CrossRef] [PubMed]

3. Wormser, G. Gamma Ray Source Working Group. Available online: http://www.eli-np.ro/documents/eli-excommeeting/ Wormser-Gamma-ray-source-working-group.pdf (accessed on 10 September 2021).

4. Hajima, R.; Shizuma, T.; Sawamura, M.; Nagai, R.; Nishimori, N.; Kikuzawa, N.; Minehara, E. First demonstration of energyrecovery operation in the JAERI superconducting linac for a high-power free-electron laser. Nucl. Instr. Meth. 2003, A507, 115-119. [CrossRef]

5. Barty, C. Development of MEGaRay Technology at LLNL. 2010. Available online: http:/ /www.eli-np.ro/executivecomitteemeeting-april-12-13.phpandprivatecommunication (accessed on 10 September 2021).

6. Brown, W.J.; Anderson, S.G.; Barty, C.P.J; Betts, S.M.; Booth, R.; Crane, J.K.; Cross, R.R.; Fittinghoff, D.N.; Gibson, D.J.; Hartemann, F.V.; et al. Experimental characterization of an ultrafast Thomson scattering X-ray source with three-dimensional time and frequency-domain analysis. Phys. Rev. 2004, 7, 060702. [CrossRef]

7. Albert, F.; Anderson, S.G.; Anderson, G.A.; Betts, S.M.; Gibson, D.J.; Hagmann, C.A.; Hall, J.; Johnson, M.S.; Messerly, M.J.; Semenov, V.A.; et al. Isotope specific detection of low-density materials with laser-based monoenergetic gamma-rays. Opt. Lett. 2010, 35, 354-356. [CrossRef] [PubMed]

8. Hajima, R. Current status and future perspectives of energy-recovery linacs. In Proceedings of the 2009 Particle Accelerator Conference, Vancouver, BC, Canada, 4-8 May 2009.

9. Neil, G.R.; Bohn, C.L.; Benson, S.V.; Biallas, G.; Douglas, D.; Dylla, H.F.; Evans, R.; Fugitt, J.; Grippo, A.; Gubeli, J.; et al. Sustained Kilowatt Lasing in a Free-Electron Laser with Same-Cell Energy Recovery. Phys. Rev. Lett. 2000, 84, 662-665. [CrossRef] [PubMed]

10. Vlase, S.; Teodorescu, P.P. Elasto-Dynamics of a Solid with a General "Rigid" Motion using FEM Model Part I. Theoretical Approach. Rom. J. Phys. 2013, 58, 872-881.

11. Gruner, S.M. Energy recovery linacs as synchrotron radiation source. Rev. Sci. Instr. 2002, 73, 1402-1406. [CrossRef] 
12. Hajima, R.; Kikuzawa, N.; Nishimori, N.; Hayakawa, T.; Shizuma, T.; Kawase, K.; Kando, M.; Minehara, E.J.; Toyokawa, H.; Ohgaki, H. Detection of radioactive isotopes by using laser Compton scattered $\gamma$-ray beams. Nucl. Instr. Meth. 2009, A608, S57-S61. [CrossRef]

13. Litvinenko, V.N.; Ben-Zvi, I.; Kayran, D.; Pogorelsky, I.; Pozdeyev, E.; Roser, T.; Yalkimenko, V. Potential uses of ERL-based gamma-ray sources. IEEE Trans. Plasma Sci. 2008, 36, 1799-1807. [CrossRef]

14. Ursescu, D.; Tesileanu, O.; Balabanski, D.; Cata-Danil, G.; Ivan, C.; Ursu, I.; Gales, S.; Zamfir, N.V. Extreme light infrastructure nuclear physics (ELI-NP): Present status and perspectives. In Proceedings of the High-Power, High-Energy, and High-Intensity Laser Technology; and Research Using Extreme Light: Entering New Frontiers with Petawatt-Class Lasers, Prague, Czech Republic, 7 May 2013; Volume 8780. [CrossRef]

15. Adriani, O.; Albergo, S.; Alesini, D.; Anania, M.; Angal-Kalinin, D.; Antici, P.; Bacci, A.; Bedogni, R.; Bellaveglia, M.; Biscari, C.; et al. Technical Design Report EuroGammaS proposal for the ELI-NP Gamma beam System. arXiv 2014, arXiv:1407.3669.

16. Perdue, B.A. Measurements of the Absolute Cross Section of the Three-Body Photodisintegration of $3 \mathrm{He}$ between $\mathrm{E}=11.4 \mathrm{MeV}$ and $14.7 \mathrm{MeV}$ at HIgammaS. Ph.D. Thesis, Duke University, Durham, NC, USA, 2010.

17. Arenhovel, H.; Sanzone, M. Photodisintegration of the Deuteron: A Review of Theory and Experiment; Springer: Vienna, Austria, 1991.

18. Blackston, M.A.; Ahmed, M.A.; Perdue, B.A.; Weller, H.R.; Bewer, B.; Pywell, R.E.; Wurtz, W.A.; Igarashi, R.; Kucuker, S.; Mikhailov, B.; et al. First observation of the splittings of the E1 p-wave amplitudes in low energy deuteron photodisintegration and its implications for the Gerasimov-Drell-Hearn Sum Rule integrand. Phys. Rev. C 2008, 78, 034003. [CrossRef]

19. Sun, C. Characterizations and Diagnostics of Compton Light Source. Ph.D. Thesis, Duke University, Durham, NC, USA, 2009.

20. Adekola, A.S.; Angell, C.T.; Hammond, S.L.; Hill, A.; Howell, C.R.; Karwowski, H.J.; Kelley, J.H.; Kwan, E. Discovery of low-lying E1 and M1 strengths in 232Th. Phys. Rev. C 2011, 83, 034615. [CrossRef]

21. Vlase, S. Elimination of Lagrangean Multipliers. Mech. Res. Commun. 1987, 14, 17-20. [CrossRef]

22. Negrean, I. New Formulations in Analytical Dynamics of Systems. Acta Tech. Napoc. Ser. Appl. Math. Mech. Eng. 2017, 60, 49-56.

23. Group, E. The White Book of ELI Nuclear Physics. Informational. 2013. Available online: https://www.nano-link.net/enewsletter_NANOPROSPECT/supliment_e-news6/ELI-NP-WhiteBook.pdf (accessed on 10 September 2021). 